\title{
Danksagung und Widmung
}

Dieses Forschungsprojekt hat sich über sechs Jahre erstreckt. Während dieser Zeit haben mich zahlreiche Menschen und Organisationen unterstützt, an die ich die folgenden Worte des Dankes adressieren möchte.

Das Projekt würde undenkbar gewesen sein ohne die Zustimmung und kontinuierliche, geduldige Unterstützung von meiner Betreuerin Frau Prof. ${ }^{\text {in }}$ Dr. Gabriele Wilde aus dem Institut für Politikwissenschaft der Universität Münster. Mit ihrer Offenheit und Präzision konnte sie mir auch in den schwierigsten Phasen zuhören und den richtigen Weg für die Entwicklung meiner Thesen weisen. Der Abgleich meines Exposees mit der finalen Version des Projekts ist für ihr Engagement der beste Beweis. Gleichzeitig möchte ich mich bei meinem zweiten Betreuer, Prof. Dr. Matthias Bohlender aus dem Institut für Sozialwissenschaften der Universität Osnabrück, für seine Bereitschaft bedanken, mir die Sichtweise auf die komplexe Problematik, die ich zu behandeln versuchte, um neue und frische Perspektiven zu erweitern. Nicht zuletzt konnte ich auch die intensive wissenschaftliche Unterstützung von Herrn Prof. Dr. Marcelo Parreira do Amaral aus dem Institut für Erziehungswissenschaft der Universität Münster während meines letzten Projektjahrs genießen und von seiner Arbeitsweise viel für meinen eigenen Forschungsstil übernehmen. All meinen Betreuern möchte ich hiermit mein tiefstes Dankgefühl ausdrücken und hoffe, ihr fachliches Renommee durch meine weitere Forschung $\mathrm{zu}$ bestätigen.

An der Graduate School of Politics (GraSP) des Instituts für Politikwissenschaft konnte ich vielen interessanten Mitarbeitern ${ }^{1}$ begegnen. An dieser Stelle möchte ich zuerst dem geschäftsführenden Direktor der GraSP, Herrn PD Dr. Matthias Freise, meinen Dank für seine Hilfsbereitschaft, seine ermutigende Zugangsweise und seinen korrekten Umgang mit all meinen Belangen ausdrücken. Des Weiteren möchte ich mich den Mitgliedern der Forschungsgruppe Gouvernementalität, namentlich Alexia, Antonia, Henrike, Christiane, Isabelle-Christine, Stephan, Stephanie, Tobias und Ulrich, für ihr engagiertes und konstruktives Interesse an mei- 
nem Forschungsthema bedanken und ihnen viel Erfolg in ihren wissenschaftlichen und persönlichen Bemühungen wünschen. Zudem möchte ich meine Dankesworte an die Freunde richten, mit denen ich das Projekt mitdiskutieren und mitschreiben konnte und die mich mit ihren Ansichten, Ratschlägen und Erfahrungen professionell und menschlich geprägt haben. Mein Dankeswort geht vor allem an Aditi, Andrea, Andrew, Deodatus, Elnur, Emmy, Christina, Janina, Sylwia, Tobias und Yimei.

Für die ersten zwei Jahre sowie in den schwierigen Überbrückungsphasen meines Projekts konnte ich mit der finanziellen Unterstützung des Deutschen Akademischen Austauschdienst (DAAD) rechnen. Die Stipendien gaben mir Sicherheit und sorgten für einen festen Boden, auf dem ich wachsen und forschen konnte. Dafür möchte ich mich besonders bedanken und mit dem erfolgreichen Ende des Projekts bezeugen, welch wichtige und relevante Rolle diesem öffentlichen Dienst zukommt.

An den Wurzeln meines Forschungsinteresses und meiner ersten Schritte in Richtung Wissenschaft standen Menschen, denen ich viel zu verdanken habe. Ich denke hier an meine Familie, für die meine Entscheidung nicht einfach war und die mich trotzdem mit allen Kräften gefördert und unterstützt hat. Ich denke an meinen besten Freund Ján, der den Mut hatte, an mich zu glauben, wo ich den Glauben an mich fast verloren hatte. Ich denke hier an meine Lektorin Heike, die für meine Vorbereitung auf den deutschen Sprachkurs Unmögliches geleistet und mich für meinen Forschungsaufenthalt mit perfekten Kenntnissen ausgestattet hatte. Ich denke hier an meinen Begleiter und Freund Ludger, der mich in alle Richtungen wachsen ließ und mich in schweren Phasen immer unterstützt hat. Ich denke hier an die Mitglieder meiner Selbsthilfegruppe, die mich mit ihrer Kraft, ihrer Erfahrung und ihrer Hoffnung bereichert und mir den Mut zur Veränderung damit geschenkt haben. Ich denke hier an meine Freundin Ursula, die mir mit ihrer Gastfreundlichkeit und ihrem netten Wort beigestanden hat. Ich denke hier an Amand und Kornelia, die bereit waren, ihre Erfahrungen mit dem Burnout-Syndrom mit mir zu teilen und mich durch ihr Engagement für eine gerechtere und liebevollere Welt $\mathrm{zu}$ inspirieren. Ich denke hier an Frau Monika Junge-Wentrup, die sich bereiterklärt hat, die Lesekorrektur meines Forschungsprojekts mit Interesse und Neugier zu übernehmen.

Zuletzt möchte ich dieses Projekt und all die Bemühungen und Krisen, die während seiner Fertigung entstanden sind, meinem Vater widmen, von dessen plötzlichem Tod ich erfuhr, als ich die ersten Zeilen des Projektentwurfs an meine Betreuerin schrieb. Die E-Mail habe ich damals gelöscht und in einem Jahr erneut geschrieben. Ich konnte seinen Tod nicht vorhersehen. Und nicht vorbeugen.

Münster, 24. Mai 2019 